\title{
Parallel Low-Complexity $M$-PSK Detector for Large-Scale MIMO Systems
}

\author{
Ganapati Hegde $^{\dagger}$, Yang Yang ${ }^{\ddagger}$, Christian Steffens ${ }^{\dagger}$, Marius Pesavento ${ }^{\dagger}$ \\ ${ }^{\dagger}$ Communication Systems Group, Technische Universität Darmstadt, Germany \\ $\ddagger$ Intel Deutschland GmbH, Germany
}

\begin{abstract}
MIMO systems have become a de-facto standard in modern wireless communication, and with the upcoming of large-scale MIMO systems, efficient symbol detection techniques become more significant than ever. In this paper, we propose a new symbol detection technique for $M$-PSK modulated symbols. In order to implement the proposed technique efficiently, we develop an iterative, low-complexity algorithm. Each iteration of the developed algorithm comprises of independent subproblems, which can be solved independently and concurrently on a parallel-computing platform, making it ideal for advanced wireless systems. Our complexity analysis reveals that the computational complexity of the proposed algorithm scales well with the number of transmit/receive antennas of the MIMO systems, and it is independent of constellation size. Simulation results show that the bit error rate (BER) of the proposed detection method is lower than those of conventional suboptimal detectors, and the BER decays faster as the size of the MIMO system increases.
\end{abstract}

\section{INTRODUCTION}

Large-scale multiple-input multiple-output (MIMO) is one of the emerging technologies capable of effectively addressing the ever increasing demand for wireless data, providing higher spectral and energy efficiency [1-3]. It is foreseen to be one of the key enablers of $5 \mathrm{G}$ technology $[4,5]$. With tens or hundreds of antennas at the transmitter and/or receiver, a large-scale MIMO system facilitates numerous data stream transmissions within the same time and frequency resources.

A main challenge associated with the large-scale MIMO technology is to design reliable and fast symbol detectors [2, 6, 7]. Even though extensive research work has been done in the last decade, and numerous techniques have been proposed [810], many of them are not well-suited for large-scale MIMO systems for two reasons. Firstly, they become infeasible or extremely slow for large systems. For example, the complexity of the optimal maximum-likelihood (ML) detector, even with its efficient implementations like the sphere decoder (SD), grows exponentially with the number of simultaneously transmitted data streams [11-13]. Secondly, suboptimal detectors such as the zero-forcing (ZF) detector, minimum mean-square error (MMSE) detector, and fixed sphere decoding (FSD), are highly sensitive to noise, and offer significantly higher bit error rates (BERs) compared with an optimal detector, in scenarios where the users' channels exhibit non-vanishing correlations [14]. Moreover, the performance of these detectors degrade further as the number of transmitted data streams approaches the number of receive antennas [15-18].

Over the last three decades, there has been tremendous advancement in semiconductor technology, with a major focus on parallel processing [19]. With the development of massively parallel processor array (MPPA) technology, it is not a distant future that we have economically viable embedded systems with hundreds or thousands of processing units on a single millimeter-size die [20-23]. Such parallel hardware architectures can benefit the large-scale MIMO systems to process a huge amount of data in real-time. By designing detection algorithms which facilitate the parallel processing, the available hardware can be efficiently utilized to speed-up the detection process.

In this paper, we propose a novel MIMO detector for $M$ PSK modulated symbols, which can be readily expressed as a convex optimization problem. To implement the proposed technique efficiently, we formulate an iterative algorithm ${ }^{1}$. Each iteration of the developed algorithm constitutes a number of subproblems, which can be solved independently and simultaneously on parallel-computing platforms, such as MPPA, to further speed up the detection process. Simulation results show that the proposed detection approach achieves significantly lower BER compared with conventional suboptimal detectors, such as the ZF and MMSE. Furthermore, the proposed scheme performs well even when the number of transmitted data streams is equal to the number of receive antennas. Theoretical analysis shows that the convergence of the proposed iterative algorithm is guaranteed, and numerical results show that it converges in a noticeably low number of iterations.

Notation: The symbol $\mathbb{C}$ denotes the set of complex numbers. Conjugate transpose operator is denoted by $(\cdot)^{H}$. The notation $\|\cdot\|$ denotes the Euclidean norm of a vector, and $|\cdot|$ denotes the absolute value of a scalar.

\section{SySTEM MODEL}

Consider a multiuser MIMO system with $N$ single antenna mobile devices simultaneously transmitting to a multiantenna base station with $K$ receive antennas, where $N \leq K$. Let $\mathbf{s}=\left[s_{1}, \ldots, s_{N}\right]^{T}$ be the transmit symbol vector, where the element $s_{n}, \forall n \in \mathcal{N} \triangleq\{1,2, \ldots, N\}$, denotes the symbol transmitted by the $n$th mobile device. Each symbol is assumed to be drawn from an $M$-ary phase-shift keying ( $M$-PSK) constellation, for $M$ being a positive integer greater than 1. Let $\mathcal{S}^{M}$ denote the set of constellation points, and $r=\left|s_{n}\right|, \forall n \in \mathcal{N}$, denote the constant amplitude of each

\footnotetext{
${ }^{1}$ After the acceptance of this paper, we found a similar approach proposed in paper [24] in the context of CDMA.
} 
constellation point. Let $\mathbf{H}=\left[\mathbf{h}_{1}, \ldots, \mathbf{h}_{N}\right] \in \mathbb{C}^{K \times N}$ be the channel matrix, with the channel vector of the $n$th mobile device denoted as $\mathbf{h}_{n} \in \mathbb{C}^{K \times 1}$. The instantaneous channel information is assumed to be perfectly known at the receiver. The received signal vector $\mathbf{y} \in \mathbb{C}^{K \times 1}$ during each symbol duration can be expressed as

$$
\mathbf{y}=\mathbf{H s}+\mathbf{n},
$$

where $\mathbf{n} \in \mathbb{C}^{K \times 1}$ is a noise vector, and all elements of the vector $\mathbf{n}$ are complex Gaussian variables with zero-mean and variance $\sigma^{2}$.

\section{Conventional Detectors}

The ML detector provides an optimal performance by performing an exhaustive search over all possible combinations of transmit symbols, i.e.,

$$
\mathbf{x}_{\mathrm{ML}}=\underset{x_{n} \in \mathcal{S}^{M}, \forall n \in \mathcal{N}}{\operatorname{argmin}}\|\mathbf{y}-\mathbf{H x}\|^{2},
$$

where $\mathrm{x}$ is a vector whose elements $x_{n}, \forall n \in \mathcal{N}$, are the optimization variables, and the vector $\mathbf{x}_{\mathrm{ML}}$ is the estimate of the transmit symbol vector $\mathbf{s}$. However, in the ML technique the search space of each transmit symbol is restricted to discrete constellation points, and its complexity grows exponentially as the number of transmit symbols increases. Therefore, the ML detector is ill-suited for the large-scale MIMO systems. On the contrary, the ZF detector completely relaxes the constraints on the transmit symbols, and searches the entire complex plane for the transmit symbol vector that minimizes the sum of squared errors, i.e.,

$$
\mathbf{x}_{\mathrm{ZF}}=\underset{x_{n} \in \mathbb{C}, \forall n \in \mathcal{N}}{\operatorname{argmin}}\|\mathbf{y}-\mathbf{H x}\|^{2} .
$$

Eq. (3) is a convex problem, and it has a closed form solution, which is given by

$$
\mathbf{x}_{\mathrm{ZF}}=\left(\mathbf{H}^{H} \mathbf{H}\right)^{-1} \mathbf{H}^{H} \mathbf{y} .
$$

In contrast to the exponential complexity of the ML detector, the ZF detector has a polynomial complexity. However, the ZF detector suffers from noise amplification in scenarios where the channel matrices have large condition numbers. The covariance matrix of the ZF estimation error vector is given by $\sigma^{2}\left(\mathbf{H}^{H} \mathbf{H}\right)^{-1}$.

\section{Proposed Symbol Detection Technique}

In this section, we propose a novel MIMO detection technique to overcome the drawbacks of the $\mathrm{ZF}$ and ML detectors.

\section{A. MIMO Detector as Convex Optimization Problem}

The ML and ZF detectors rest on the opposite extremes on the transmit symbol search space scale; the ML detector search space is limited to the discrete constellation points, and in contrast, the ZF detector search space occupies the entire complex plain. We propose to relax the search space constraints on the transmit symbols from the discrete points to a closed disk space, with the $M$-PSK constellation points residing on the boundary of the disk. The benefits of limiting the search space to the disk space are twofold. Firstly, due

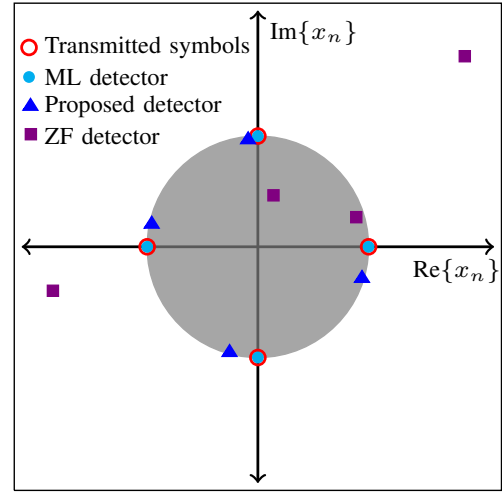

Fig. 1: Solutions of different detectors for a $4 \times 4$ MIMO system with 4-PSK modulation scheme. The gray region represents the search space of the proposed detection method.

to the restriction on the search space the noise amplification is bounded (see Fig. 1), and thus the BER will be reduced compared with the ZF detector. Secondly, the disk is a convex set, which enable us to formulate the detection problem as the following simple convex optimization problem:

$$
\begin{aligned}
\mathbf{x}^{*}= & \underset{x_{n}, \forall n \in \mathcal{N}}{\operatorname{argmin}}\|\mathbf{y}-\mathbf{H x}\|^{2} \\
& \text { subject to }\left|x_{n}\right|^{2} \leq r^{2}, \quad \forall n \in \mathcal{N} .
\end{aligned}
$$

Similar to the ZF detector, elementary slicing (quantization) operation can be performed on $\mathrm{x}^{*}$ to obtain the estimated symbols.

\section{B. Parallel Low-Complexity Iterative Algorithm}

In this subsection, we develop an efficient iterative algorithm to solve problem (5) in a parallel, distributed manner, to accelerate the detection process in a large-scale MIMO system equipped with multiple parallel processing units.

The presentation of the new algorithm is organized as follows. Firstly, an iterative approximate problem is formulated to the original convex optimization problem, whose convergence is guaranteed to the optimal solution of the original problem. Then, the approximate problem is decomposed into $N$ independent subproblems, which can be solved in parallel. Subsequently, a closed form solution to the subproblems is constructed. Computation of the optimal step-size, as required for the update phase, is presented afterwards.

The Approximate Problem: In order to tailor problem (5) for the parallel implementation, we decompose the problem into multiple subproblems, which can be solved independently and simultaneously. To achieve this goal, we exploit the fact that the objective function in (5a) of problem (5) is convex in each variable $x_{n}, \forall n \in \mathcal{N}$. Employing the Jacobi algorithm [25-27], we can formulate the approximate function of the original objective function $f(\mathbf{x})=\|\mathbf{y}-\mathbf{H x}\|^{2}$, whose convergence to the optimal solution of the original problem is ensured with an appropriate step-size selection. Let $\mathbf{x}^{t}$ denote the approximate solution to the problem (5) obtained in the $(t-1)$ th iteration. At $t=0, \mathbf{x}^{0}$ is assigned with any random feasible vector. Based on the Jacobi algorithm, the 
approximate function of $f(\mathbf{x})$ in the $t$ th iteration around the point $\mathbf{x}^{t}$ can be expressed as

$$
\sum_{n=1}^{N}\left\|\mathbf{y}-\mathbf{H}_{-n} \mathbf{x}_{-n}^{t}-\mathbf{h}_{n} x_{n}\right\|^{2} .
$$

In Eq. (6), $\mathbf{H}_{-n}$ denotes the matrix obtained by eliminating the $n$th column $\mathbf{h}_{n}$ from matrix $\mathbf{H}$, and $\mathbf{x}_{-n}$ denotes the vector obtained by discarding the $n$th element $x_{n}$ from vector $\mathbf{x}$.

Upon defining $\mathbf{y}_{-n}^{t} \triangleq \mathbf{y}-\mathbf{H}_{-n} \mathbf{x}_{-n}^{t}$, the resulting approximate problem can be expressed as

$$
\begin{aligned}
\hat{\mathbf{x}}^{t}=\underset{x_{n}, \forall n \in \mathcal{N}}{\operatorname{argmin}} \sum_{n=1}^{N}\left\|\mathbf{y}_{-n}^{t}-\mathbf{h}_{n} x_{n}\right\|^{2} \\
\quad \text { subject to }\left|x_{n}\right|^{2} \leq r^{2}, \quad \forall n \in \mathcal{N} .
\end{aligned}
$$

The vector $\hat{\mathbf{x}}^{t}-\mathrm{x}^{t}$ represents a descent direction of the objective function $f(\mathbf{x})$ in the domain of problem (5) [26]. Therefore, the vector $\mathbf{x}^{t}$ is updated after each iteration, using the following rule:

$$
\mathbf{x}^{t+1}=\mathbf{x}^{t}+\gamma^{t}\left(\hat{\mathbf{x}}^{t}-\mathbf{x}^{t}\right)
$$

where $\gamma^{t} \in(0,1]$ is an appropriate step-size. The algorithm has converged to the optimal solution $\mathbf{x}^{*}$, when $\hat{\mathbf{x}}^{t}=\mathbf{x}^{t}$.

Decomposition of the Approximate Problem: The objective function in (7a) of problem (7) is composed of $N$ addends, where each addend involves only one variable $x_{n}, \forall n \in \mathcal{N}$. Furthermore, the constraint set (7b) is a Cartesian product of closed convex sets, with each convex set corresponds to one variable $x_{n}, \forall n \in \mathcal{N}$. Consequently, we can decompose problem (7) into $N$ independent subproblems [27] as

$$
\begin{aligned}
\hat{x}_{n}= & \underset{x_{n}}{\operatorname{argmin}}\left\|\mathbf{y}_{-n}^{t}-\mathbf{h}_{n} x_{n}\right\|^{2} \\
& \text { subject to }\left|x_{n}\right|^{2} \leq r^{2},
\end{aligned}
$$

where $\hat{x}_{n}$ is the $n$th element of vector $\hat{\mathbf{x}}^{t}$. Since each subproblem exclusively depends on a single variable, each subproblem can be solved independently and concurrently.

Closed Form Solution of the Subproblem: In order to derive a closed form expression for the optimal solution $\hat{x}_{n}$ of the convex problem (9), we apply the Lagrangian duality principle, and derive the Karush-Kuhn-Tucker (KKT) optimality system [28].

The Lagrangian function $L\left(x_{n}, \lambda_{n}\right)$ associated with problem (9) can be written as

$$
L\left(x_{n}, \lambda_{n}\right)=\left\|\mathbf{y}_{-n}^{t}-\mathbf{h}_{n} x_{n}\right\|^{2}+\lambda_{n}\left(\left|x_{n}\right|^{2}-r^{2}\right),
$$

where $\lambda_{n}$ is a Lagrangian multiplier. Let $\hat{x}_{n}$ and $\hat{\lambda}_{n}$ be any primal and dual optimal points with zero duality gap. Then, we have the following KKT conditions:

$$
\begin{array}{lr}
\hat{x}_{n}=\left(\left\|\mathbf{h}_{n}\right\|^{2}+\hat{\lambda}_{n}\right)^{-1} \mathbf{h}_{n}^{H} \mathbf{y}_{-n}^{t}, & \text { (stationarity) } \\
\left|\hat{x}_{n}\right|^{2}-r^{2} \leq 0, & \text { (primal feasibility) } \\
\hat{\lambda}_{n} \geq 0, & \text { (dual feasibility) } \\
\hat{\lambda}_{n}\left(\left|\hat{x}_{n}\right|^{2}-r^{2}\right)=0 . & \text { (complementary slackness) }
\end{array}
$$

There are two mutually exclusive possibilities that arise due to Eq. (11c): a) $\hat{\lambda}_{n}=0$, b) $\hat{\lambda}_{n}>0$. Suppose $\hat{\lambda}_{n}=0$, using
Eq. (11a), the corresponding value of $x_{n}$ can be expressed as

$$
x_{n}^{\left(\hat{\lambda}_{n}=0\right)}=\frac{\mathbf{h}_{n}^{H} \mathbf{y}_{-n}^{t}}{\left\|\mathbf{h}_{n}\right\|^{2}} .
$$

If $x_{n}^{\left(\hat{\lambda}_{n}=0\right)}$ satisfies Eq. (11b), then all the KKT conditions are fulfilled, and $\hat{x}_{n}=x_{n}^{\left(\hat{\lambda}_{n}=0\right)}$. However, if $x_{n}^{\left(\hat{\lambda}_{n}=0\right)}$ does not satisfy Eq. (11b), then $\hat{\lambda}_{n}>0$ must hold. Moreover, due to Eq. (11d), we have

$$
\left|\hat{x}_{n}\right|^{2}-r^{2}=0 \text {. }
$$

Substituting Eq. (11a) into Eq. (13), we obtain

$$
\left(\mathbf{y}_{-n}^{t}\right)^{H} \mathbf{h}_{n}\left(\left\|\mathbf{h}_{n}\right\|^{2}+\hat{\lambda}_{n}\right)^{-2} \mathbf{h}_{n}^{H} \mathbf{y}_{-n}^{t}-r^{2}=0 .
$$

Solving Eq. (14), $\hat{\lambda}_{n}(>0)$ can be derived as

$$
\hat{\lambda}_{n}=\frac{\left|\mathbf{h}_{n}^{H} \mathbf{y}_{-n}^{t}\right|}{r}-\left\|\mathbf{h}_{n}\right\|^{2} \text {. }
$$

Finally, substituting Eq. (15) into Eq. (11a), we obtain

$$
x_{n}^{\left(\hat{\lambda}_{n}>0\right)}=r \frac{\mathbf{h}_{n}^{H} \mathbf{y}_{-n}^{t}}{\left|\mathbf{h}_{n}^{H} \mathbf{y}_{-n}^{t}\right|} .
$$

Eq. (16) can be interpreted as the projection of the point $\mathbf{h}_{n}^{H} \mathbf{y}_{-n}^{t}$ onto the disk of radius $r$, and the technique is known as the gradient projection method [29, 30].

Combining Eq. (12) and Eq. (16), the optimal solution of problem (9) can be mathematically expressed as

$$
\hat{x}_{n}=\min \left(\frac{\left|\mathbf{h}_{n}^{H} \mathbf{y}_{-n}^{t}\right|}{\left\|\mathbf{h}_{n}\right\|^{2}}, r\right) \frac{\mathbf{h}_{n}^{H} \mathbf{y}_{-n}^{t}}{\left|\mathbf{h}_{n}^{H} \mathbf{y}_{-n}^{t}\right|} .
$$

Computation of Optimal Step-size: The optimal update step-size $\gamma^{t}$ in Eq. (8), which minimizes the objective function $f(\mathbf{x})$ in the domain of problem (5), can be computed using the exact line search method $[26,30]$ as

$$
\gamma^{t}=\underset{\gamma \leq 1}{\operatorname{argmin}} \underbrace{\left\|\mathbf{y}-\mathbf{H}\left(\mathbf{x}^{t}+\gamma\left(\hat{\mathbf{x}}^{t}-\mathbf{x}^{t}\right)\right)\right\|^{2}}_{Z(\gamma)} .
$$

Since $Z(\gamma)$ in Eq. (18) is a convex function in $\gamma$, and $\hat{\mathbf{x}}^{t}-\mathbf{x}^{t}$ is a descent direction, $\gamma^{t}$ is always non-negative. Moreover, suppose the gradient of $Z(\gamma)$ with respect to $\gamma$ vanishes at $\gamma^{*}$, then $\gamma^{t}$ is the projection of $\gamma^{*}$ onto the interval $[0,1]$. Consequently, $\gamma^{t}$ can be computed as

$$
\gamma^{t}=\left[\frac{\operatorname{Re}\left\{\mathbf{p}^{H} \mathbf{q}\right\}}{\mathbf{q}^{H} \mathbf{q}}\right]_{0}^{1},
$$

where $\mathbf{p}=\mathbf{y}-\mathbf{H} \mathbf{x}^{t}, \mathbf{q}=\mathbf{H}\left(\hat{\mathbf{x}}^{t}-\mathbf{x}^{t}\right)$, and $[\cdot]_{0}^{1}$ denotes the projection operation onto the interval $[0,1]$. Furthermore, the exact line search based step-size computation according to Eq. (19) guarantees the convergence of Eq. (8) to the optimal solution of problem (5) [27, 30].

In summary, each iteration of the proposed algorithm consists of computing solutions of each subproblem according to Eq. (17), computing the corresponding step-size according to Eq. (19), and performing the update according to Eq. (8).

\section{Computational Complexity Analysis}

In this subsection, we analyse the complexity of the proposed algorithm, in terms of complex multiplications (CMs) 


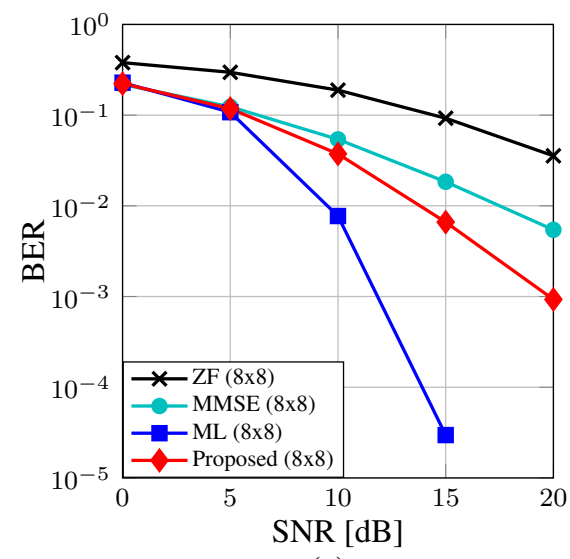

(a)

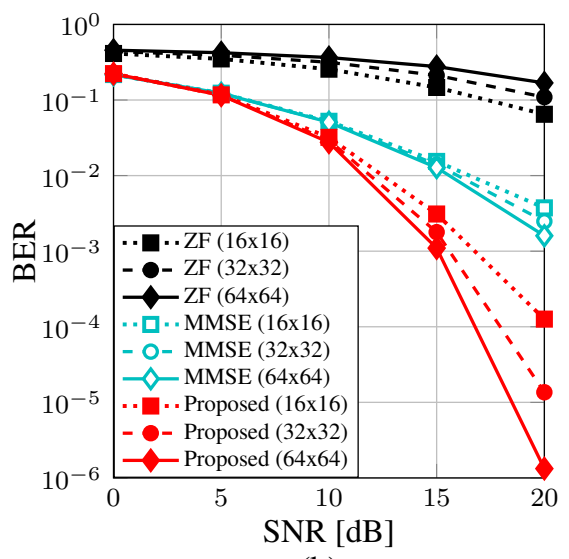

(b)

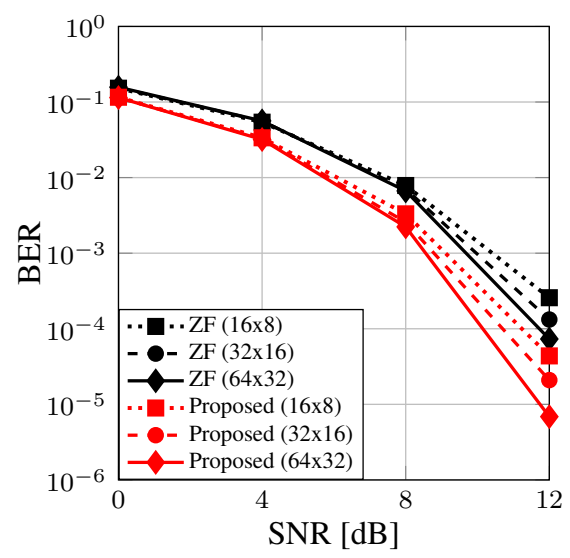

(c)

Fig. 2: BER vs. SNR of various detectors on MIMO systems of different orders for a 4-PSK modulation scheme.

and complex additions (CAs).

The terms $\mathbf{h}_{n}^{H} \mathbf{h}_{n}, \mathbf{h}_{n}^{H} \mathbf{y}$, and $\mathbf{h}_{n}^{H} \mathbf{H}_{-n}$ can be computed concurrently, $\forall n \in \mathcal{N}$, in the initiation phase of the algorithm. The only major computation involved in each subproblem of an iteration is the calculation of $\left(\mathbf{h}_{n}^{H} \mathbf{H}_{-n}\right) \mathbf{x}_{-n}^{t}$, which is needed for the computation of $\mathbf{h}_{n}^{H} \mathbf{y}_{-n}^{t}$. The approximate numbers of CMs and CAs involved in the initiation phase and in each iteration are listed in Table I. The proposed algorithm has a complexity that is cubic with the dimension of $\mathbf{H}$, similar to the ZF and MMSE detectors [31]. Moreover, on a parallelcomputing platform with $N$ parallel processors, the initiation phase and the step-size computation phase of the proposed algorithm have quadratic complexities, and each subproblem and update-phase have linear complexities on each processor.

\section{Numerical RESUlts}

In this section, we compare the performance of the proposed detection technique and the conventional detection techniques, namely, the ZF detector, the MMSE detector, and the ML detector, in the case of 4-PSK modulation.

Fig. 2-(a) shows that the BER achieved by the proposed detection method is significantly lower than that of the ZF detector and MMSE detector. In Fig. 2-(b), it can be noticed that the proposed detection method performs well even when the number of transmitted symbols $N$ is equal to the number of receive antennas $K$, and the BER reduces further as the size of the MIMO system grows. On the contrary, the BER of the ZF detector in such systems is significantly higher, and its BER increases further for higher order MIMO systems due to the noise amplification effects. The performance improvement of the proposed detector results from the increase in the number of constraints of problem (5), for higher order MIMO systems. Fig. 2-(c) shows that the performance of the proposed method is considerably better than the ZF detector even after reducing the number of transmitted symbols $N$ to the half of the number of receive antennas $K$.

Fig. 3 shows that low iteration numbers are sufficient to achieve the optimal BER of the proposed detection scheme, especially when $\mathrm{SNR} \leq 10 \mathrm{~dB}$.

In Table II we can notice that, in the SNR range of 0-20 $\mathrm{dB}$, only 2 iterations are sufficient for the proposed algorithm

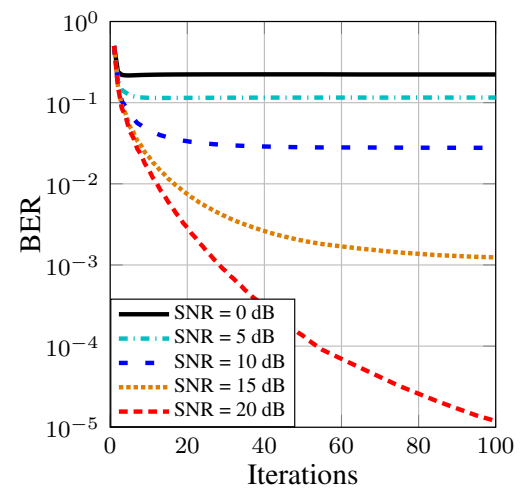

Fig. 3: BER vs. iterations of the proposed detection method for a $64 \times 64$ MIMO system with 4-PSK modulation scheme.

TABLE I: Approximate number of CMs and CAs required to implement the proposed algorithm.

\begin{tabular}{|c|c|c|}
\hline- & CMs & CAs \\
\hline Initiation phase & $N K(N+1)$ & $N(N+1)(K-1)$ \\
\hline Per iteration & $N^{2}+2 N K+2 K$ & $N^{2}+2 N K+N+K$ \\
\hline
\end{tabular}

to deliver the BER performance of ZF detector on a $64 \times 64$ MIMO system, and less than 25 iterations are sufficient to match the performance of the MMSE detector.

TABLE II: Number of iterations required by the proposed algorithm to match the BER performance of the ZF and MMSE detectors on a $64 \times 64$ MIMO system.

\begin{tabular}{|l|c|c|c|c|c|}
\hline SNR in dB & 0 & 5 & 10 & 15 & 20 \\
\hline ZF detector & 2 & 2 & 2 & 2 & 2 \\
\hline MMSE detector & 5 & 6 & 9 & 15 & 24 \\
\hline
\end{tabular}

\section{CONCLUSION}

A novel MIMO detection technique has been proposed, and formulated as a convex optimization problem. To speed up the detection process, a parallel, low-complexity, iterative algorithm has been developed. Numerical results show that the BER performance of the proposed detection approach is better than that of the conventional suboptimal detectors. The performance of the proposed technique further inflates as the order of the MIMO system increases. 


\section{REFERENCES}

[1] E. Larsson, O. Edfors, F. Tufvesson, and T. Marzetta, "Massive MIMO for next generation wireless systems," IEEE Commun. Mag., vol. 52, no. 2, pp. 186-195, Feb. 2014.

[2] F. Rusek, D. Persson, B. K. Lau, E. G. Larsson, T. L. Marzetta, O. Edfors, and F. Tufvesson, "Scaling up MIMO: Opportunities and challenges with very large arrays," IEEE Signal Process. Mag., vol. 30, no. 1, pp. 40-60, Jan. 2013.

[3] J. Hoydis, S. T. Brink, and M. Debbah, "Massive MIMO in the UL/DL of cellular networks: How many antennas do we need?," IEEE J. Select. Areas Commun., vol. 31, no. 2, pp. 160-171, Feb. 2013.

[4] J. G. Andrews, S. Buzzi, W. Choi, S. V. Hanly, A. Lozano, A. C. K. Soong, and J. C. Zhang, "What will 5G be?," IEEE J. Select. Areas Commun., vol. 32, no. 6, pp. 1065-1082, Jun. 2014.

[5] F. Boccardi, R. W. Heath, A. Lozano, T. L. Marzetta, and P. Popovski, "Five disruptive technology directions for 5G," IEEE Commun. Mag., vol. 52, no. 2, pp. 74-80, Feb. 2014

[6] L. Lu, G. Y. Li, A. L. Swindlehurst, A. Ashikhmin, and R. Zhang, "An overview of massive MIMO: Benefits and challenges," IEEE J. Select. Topics in Signal Processing, vol. 8, no. 5, pp. 742-758, Oct. 2014.

[7] R. C. D. Lamare, "Massive MIMO systems: Signal processing challenges and research trends," CoRR, 2013.

[8] E. G. Larsson, "MIMO detection methods: How they work," IEEE Signal Process. Mag., vol. 26, no. 3, pp. 91-95, May 2009.

[9] A. B. Gershman and N. D. Sidiropoulos, Space-time processing for MIMO communications, John Wiley \& Sons, 2005.

[10] L. Bai and J. Choi, Low Complexity MIMO Detection, Springer New York, 2012.

[11] E. Viterbo and J. Boutros, "A universal lattice code decoder for fading channels," IEEE Trans. Inform. Theory, vol. 45, no. 5, pp. 1639-1642, Jul. 1999.

[12] M. O. Damen, H. E. Gamal, and G. Caire, "On maximum-likelihood detection and the search for the closest lattice point," IEEE Trans. Inform. Theory, vol. 49, no. 10 , pp. 2389-2402, Oct. 2003.

[13] J. Jalden and B. Ottersten, "On the complexity of sphere decoding in digital communications," IEEE Trans. Signal Processing, vol. 53, no. 4, pp. 1474-1484, Apr. 2005.

[14] H. Artes, D. Seethaler, and F. Hlawatsch, "Efficient detection algorithms for MIMO channels: A geometrical approach to approximate ML detection," IEEE Trans. Signal Processing, vol. 51, no. 11, pp. 2808-2820, Nov. 2003.

[15] L. G. Barbero and J. S. Thompson, "Fixing the complexity of the sphere decoder for MIMO detection," IEEE Trans. Wireless Commun., vol. 7, no. 6, pp. 2131-2142, Jun. 2008.
[16] D. W. Waters and J. R. Barry, "The chase family of detection algorithms for multiple-input multiple-output channels," IEEE Trans. Signal Processing, vol. 56, no. 2, pp. 739-747, Feb. 2008.

[17] A. Elghariani and M. Zoltowski, "Low complexity detection algorithms in large-scale MIMO systems," IEEE Trans. Wireless Commun., vol. PP, no. 99, pp. 1-1, Oct. 2015.

[18] P. Li and R. D. Murch, "Multiple output selection-LAS algorithm in large MIMO systems," IEEE Communications Letters, vol. 14, no. 5, pp. 399-401, May 2010.

[19] H. El-Rewini and M. Abd-El-Barr, Advanced computer architecture and parallel processing, John Wiley \& Sons, 2005.

[20] K. Asanovic, R. Bodik, B. C. Catanzaro, J. J. Gebis, P. Husbands, K. Keutzer, D. A. Patterson, W. L. Plishker, J. Shalf, S. W. Williams, and K. A. Yelick, "The landscape of parallel computing research: A view from Berkeley," Tech. Rep. UCB/EECS-2006-183, EECS Department, University of California, Berkeley, Dec. 2006.

[21] S. Borkar, "Thousand core chips: A technology perspective," in Proc. 44th Annual Design Automation Conf. (DAC), San Diego, CA, USA, Jun. 2007, pp. 746-749, ACM.

[22] D. B. Kirk and W. W. Hwu, Programming Massively Parallel Processors: A Hands-on Approach, Morgan Kaufmann Publishers Inc., San Francisco, CA, USA, 1st edition, 2010

[23] G. G. Pechanek, S. Vassiliadis, and J. G. Delgado-Frias, "Massively parallel array processor," Jun. 2002, US Patent 6,405,185.

[24] P. H. Tan, L. K. Rasmussen, and T. J. Lim, "Constrained maximum-likelihood detection in CDMA," IEEE Trans. Commun., vol. 49, no. 1, pp. 142-153, Jan. 2001.

[25] G. Scutari, F. Facchinei, P. Song, D. P. Palomar, and J. S. Pang, "Decomposition by partial linearization: Parallel optimization of multi-agent systems," IEEE Trans. Signal Processing, vol. 62, no. 3, pp. 641-656, Feb. 2014.

[26] Y. Yang and M. Pesavento, "A novel line search method for nonsmooth optimization problems," in Proc. 23th European Signal Processing Conf. (EUSIPCO), Nice, France, Aug. 2015, pp. 1756-1760.

[27] Y. Yang and M. Pesavento, "A novel iterative convex approximation method," arXiv preprint arXiv:1506.04972, Jun. 2015, (submitted to IEEE Trans. Signal Processing).

[28] S. Boyd and L. Vandenberghe, Convex Optimization, Cambridge University Press, New York, NY, USA, 2004.

[29] D. G. Luenberger, Introduction to linear and nonlinear programming, Addison-Wesley Reading, MA, 1973.

[30] D. P. Bertsekas, Nonlinear programming, Athena scientific, 2nd edition, 1999.

[31] K. L. Du and M. N. S. Swamy, Wireless Communication Systems: From RF Subsystems to $4 G$ Enabling Technologies, Cambridge University Press, 2010. 